\title{
The outcomes of home treatment for schizophrenia
}

\author{
Hannah Chu Han Huang, ${ }^{1}$ Mark Taylor, ${ }^{2,3,4}$ Alasdair Carmichael $^{2}$
}

BJPsych Bulletin (2018) 42, 238-242, doi:10.1192/bjb.2018.56

\begin{abstract}
${ }^{1}$ South London and Maudsley Trust: ${ }^{2}$ University of Edinburgh; ${ }^{3}$ University of Queensland; ${ }^{4}$ Metro South Health, Brisbane

Correspondence to M. Taylor (marktaylor2@nhs.net)

First received 25 Aug 2017, final revision 25 Apr 2018, accepted 1 Jun 2018

(C) The Authors 2018. This is an Open Access article, distributed under the terms of the Creative Commons Attribution-NonCommercial-ShareAlike licence (http://creativecommons.org/ licenses/by-nc-sa/4.0/), which permits non-commercial re-use, distribution, and reproduction in any medium, provided the same Creative Commons licence is included and the original work is properly cited. The written permission of Cambridge University Press must be obtained for commercial re-use.
\end{abstract}

\begin{abstract}
Aims and method We examined whether intensive home treatment (IHTT) was beneficial for acute schizophrenia, using the Clinical Global Impression (CGI) scale as a measure of severity and change, between 2011-2015. Demographic and clinical details were collected.
\end{abstract}

Results 309 cases were referred to IHTT, comprising 245 separate individuals. This represented all severe acute psychotic episodes in Edinburgh during the study period. Three quarters of individuals had an improvement in CGI following IHTT and were safely managed at home. Thirty-nine per cent of all people received three or more regular medications. Comorbid drug and alcohol misuse was also frequently seen.

Clinical implications IHTT can be beneficial to those suffering an acute episode of psychosis and has been shown to improve overall clinical outcome based on the CGI. Medication polypharmacy, as well as drug and alcohol use, are commonly seen in this population.

Declaration of interest M.T. worked in IHTT at the time of the study, and has received fees and/or hospitality from Janssen, Lundbeck and Otsuka in the past 3 years.

Keywords Schizophrenia; outcome studies; community mental health teams.

\section{Background}

Crisis resolution and home treatment (CRHT) teams have been recommended in mental health for many years, ${ }^{1}$ with intensive $24 \mathrm{~h}$ home treatment being seen as an alternative to hospital admission. A randomised control trial reported by Johnson et $a l^{1}$ showed a reduction in the number of hospital admissions as well as a decrease in the cost to the service $^{2}$ with CRHT. There have been positive experiences for patients or consumers of $\mathrm{CRHT}^{3}$ although reports have also highlighted some dissatisfaction, namely that too many staff members are involved and the contacts are short-lived. ${ }^{4}$

The Edinburgh CRHT is called the Intensive Home Treatment Team (IHTT) ${ }^{3}$ and has two main points of entry into treatment - referral from the community (either from primary or secondary care) and from in-patient units to facilitate early discharge from hospital (but no selfreferral). Edinburgh has well developed community mental health teams but no specific first episode or early intervention programme for adult psychosis; therefore, as far as is known, all acutely relapsing patients with schizophrenia and psychosis are referred to the IHTT.

Coping with schizophrenia and related psychoses remains a challenge, and globally ${ }^{5}$ schizophrenia contributes $7.4 \%$ of all lost years of life to all disabilities. Relapse of schizophrenia, sadly, is common, with only about $20 \%$ of patients having only one episode of psychosis. ${ }^{6,7}$ Predictors of relapse include medication non-adherence and comorbid substance misuse, ${ }^{6,7}$ and relapse is considered a time of increased risk of suicide in schizophrenia. ${ }^{8}$

\begin{abstract}
Aims
Although there have been reports of patient satisfaction with CRHTs, very few studies have examined how a CRHT affects patients' clinical outcomes. In this observational study, we examine whether intensive home treatment was of benefit to people experiencing a relapse of schizophrenia in terms of routine clinical outcome measures, and identify the demographic and clinical characteristics of those who have responded (or not) to this intervention.
\end{abstract}

\section{Method}

Data were collected retrospectively from medical records of patients assessed and cared for by the IHTT between 2011 and 2015. Those with an ICD-10 code diagnosis of schizophrenia (F20) and related disorders (F22 - delusional disorder, F23 - acute transient psychosis, F25 - schizoaffective disorder, F28 - other psychotic disorder not due to substance or known physiological condition, and F29 unspecified psychosis not due to a substance or known physiological condition) were included. The validity of the diagnosis was established by consultant psychiatrist review. Using unique identifying codes, the electronic records of these patients were retrieved and reviewed using a priori criteria. Ethical approval for the study was provided by the University of Edinburgh, UK.

IHTT records the severity of the presenting mental disorder using the Clinical Global Impression Scale ${ }^{9}$ (CGI) at the time of referral to IHTT. The CGI objectively assesses 
the global severity of illness and change in clinical condition over time. ${ }^{9}$ CGI-S is rated on the following seven-point scale: 1 , normal/not at all ill; 2 , borderline mentally ill; 3 , mildly ill; 4, moderately ill; 5, markedly ill; 6 , severely ill; 7, among the most extremely ill patients. CGI-I is similarly rated on a seven-point scale: 'compared with the patient's condition at admission, this patient's condition is' 1, very much improved: 2, much improved; 3, minimally improved; 4, no change; 5 , minimally worse; 6 , much worse; 7 , very much worse. IHTT clinicians noted the improvement or lack thereof in the presenting condition via the CGIImprovement (CGI-I) score at the point of discharge from IHTT. This was routinely rated for every patient during his or her care with the team by an adequately trained clinician, although the pattern of staff shift working meant that the CGI and CGI-I for an individual patient were not always rated by the same clinician.

Other data collected for each patient included demographic details and relevant clinical details (see Supplementary Appendix 1 available at https://doi.org/10. 1192/bjb.2018.56 for full list), as well as data about each patient's regular medication(s) (see Supplementary Appendix 2 for a list of categories).

\section{Statistical analysis}

Unpaired $t$-tests were used (with the assumption of normality in CGI distribution being satisfied) to test whether there was a statistically significant change in CGI for patients who were discharged to the community, compared with those who required hospital admission, as well as to determine whether there was a statistically significant reduction in CGI for all referrals treated by IHTT.

Pearson's $\chi^{2}$-test was used to test the hypothesis that people who were not prescribed medication or were nonadherent with their medication were more likely to be admitted to hospital than patients who were taking prescribed medication.

Mixed model regression analysis was used to control for any confounders that could be significantly associated with CGI difference. Variables included as potential confounders were age, gender, treatment duration, number of psychotropic medications prescribed, and drug and alcohol status. Pearson's correlation was also conducted to see whether there was a correlation between CGI and age or gender.

\section{Results}

\section{Demographics of patient population}

Over the 5-year period, there were 309 referrals to IHTT that met the inclusion criteria, comprising 245 separate individuals with the majority (82\%) being new to IHTT and mental health services. Thirty-six individuals were referred more than once, with 16 people being referred to IHTT at least three times. One person was referred on five separate occasions, and another individual was referred seven different times. The patients referred had a mean age of 41 years, and 162 (52\%) were men (Table 1).

\begin{tabular}{|lc|}
\hline Table 1 & \multicolumn{1}{l}{ Demographics of individual referrals } \\
& $N=309$ \\
\hline Age, mean (range), S.D & $40.4(18-66), 12.0$ \\
\hline Male gender, $n$ (\%) & $158(51)$ \\
\hline $\begin{array}{l}\text { Number of psychotropic medications } \\
\text { prescribed, mean (range), S.D }\end{array}$ & $1.6(0-4), 1.0$ \\
\hline Treatment duration in days, mean (range), S.D & $22.3(1-167), 23.2$ \\
\hline Drug use: $n$ (\%) & $23(7.4)$ \\
\hline Alcohol use: $n$ (\%) & $46(14.9)$ \\
\hline Both drug and alcohol use: $n$ (\%) & $26(8.4)$ \\
\hline
\end{tabular}

\section{Referrals and outcomes}

The majority of the 309 referrals to IHTT over the 5-year period $(56 \%, n=173)$ were from the community. The remaining $44 \%$ of referrals were from in-patient units, in order to facilitate early discharge from hospital.

In terms of outcomes at discharge from IHTT, a total of 221 referrals $(76 \%)$ were discharged to the community, and 70 referrals (24\%) required transfer into hospital owing to their risk status or severity of illness. Twenty-seven per cent of repeat referrals were discharged to hospital, compared with $23 \%$ of 'one-off' referrals ( $P$-value $=0.6$ ).

There were five deaths $(2 \%)$ within the 5 -year period, including one suicide, from the total group of 255 people. None of those who died were under the care of IHTT at the time of death. Two of the deceased had a history of extensive alcohol and drug misuse. All five of those who died had a history of repeated hospital admissions. The suicide occurred in December 2013, after an urgent referral to hospital, but the person was not deemed unwell enough for admission at that time.

Fifty-two per cent (14 referrals) of the 27 referrals who were on no regular medications, or who were non-adherent with their medications, went on to require hospital admission, in comparison with $20 \%$ of patients who were taking at least one medication, and $24 \%$ of all referrals.

Eighteen referral outcomes were missing from records throughout the 5-year period.

\section{CGI}

The mean value of CGI of all patients at admission to IHTT was 4.1. The mean value of CGI of all patients at discharge was 3.05, an improvement of 1.05 (Table 2), which was a significant change $(P<0.05)$. Patients who were later admitted to hospital from IHTT had a higher mean value of CGI at 4.2 on admission to IHTT compared with the mean value of CGI of all patients. The mean value of CGI on discharge to hospital in this population was 4.6, i.e. they were more unwell, according to CGI.

Patients whose clinical outcome was discharge back to their usual carers had a mean value of CGI of 4.04 on admission to IHTT and a mean value of CGI on discharge from IHTT of 2.57.This was a decrease of mean CGI (i.e. improvement in health) of 1.47 . 


\begin{tabular}{|c|c|c|c|c|c|c|}
\hline Year & $\begin{array}{c}2011 \\
(n=42)\end{array}$ & $\begin{array}{c}2012 \\
(n=33)\end{array}$ & $\begin{array}{c}2013 \\
(n=60)\end{array}$ & $\begin{array}{c}2014 \\
(n=80)\end{array}$ & $\begin{array}{c}2015 \\
(n=94)\end{array}$ & $\begin{array}{c}\text { Total } \\
(n=309)\end{array}$ \\
\hline Average CGI at referral - all patients & 3.8 & 4.2 & 4.3 & 4.0 & 4.1 & 4.1 \\
\hline Average CGI on discharge - all patients & 3.3 & 3.0 & 2.9 & 3.3 & 2.8 & 3.1 \\
\hline Average CGI at referral, those later discharged to the community & 3.8 & 4.2 & 4.2 & 3.9 & 4.1 & 4.0 \\
\hline Average CGI on discharge to the community & 3.0 & 2.9 & 2.5 & 2.7 & 2.2 & 2.6 \\
\hline Average CGI at referral, later transferred to hospital & 3.8 & 4.3 & 4.6 & 4.3 & 4.1 & 4.2 \\
\hline Average CGI on transfer to hospital & 3.5 & 3.25 & 4.2 & 5.0 & 5.0 & 4.6 \\
\hline
\end{tabular}

The difference in average CGI between the mean discharge CGI of all referrals and the mean discharge value of patients admitted to hospital was non-significant (1.55, $P<$ 0.15). The difference in average CGI, on discharge, between referrals admitted to hospital and referrals discharged to the community was 2.03 , a statistically significant change $(P<0.05)$.

The average CGI of all repeat referrals at the beginning of treatment with IHTT was 3.94, 0.11 lower than the average CGI of all referrals. The CGI on discharge for repeat referrals was 3.22 , an average decrease in CGI of 0.72 . These differences were not significant.

Age, gender, number of medications prescribed and drug/alcohol status were found not to be significant confounders in the regression analysis (see Supplementary Appendix 3). Only treatment duration was found to be a significant confounder, with patients having a longer treatment duration demonstrating a greater difference in CGI.

\section{Medication}

Table 3 illustrates the different medications and number of patients taking them: $2.8 \%$ of patients were on no medications, $31 \%$ were prescribed one medication, $27 \%$ were prescribed two medications, $18 \%$ were prescribed three medications and $21 \%$ were prescribed four or more medications. The mean number of any medications, including nonpsychotropic medications, taken by an average patient across the 5 years was 2.1. A total of 27 referrals (9\%) refused medication, did not require medication or were judged as non-adherent.

Pearson's $\chi^{2}$-test showed that patients who were taking no medications, or were non-concordant with their medication, were more likely to be admitted to hospital from IHTT compared with patients who were taking at least one medication $\left(\chi^{2}=43.3\right)$

\section{Drug and alcohol use}

In total, 122 (39.5\%) referrals were misusing either alcohol or drugs (Table 4) as rated by the IHTT clinician. The drug use was varied and often involved poly-substance misuse. Cannabis had the highest frequency of use, followed by amphetamines.

\section{Discussion}

Crisis resolution and home treatment teams, such as IHTT, are designed to manage acute mental health problems in the individual's home environment, minimising the risk of hospital admission while promoting autonomy and self-efficacy. It is incumbent on health services to demonstrate the effectiveness of their interventions, and IHTT from inception chose the CGI as an observer-rated measure of illness severity and subsequent improvement (or deterioration).

Our results show that $76 \%$ of people were discharged back to their usual care after IHTT involvement following

Table 3 Medications prescribed

\begin{tabular}{|c|c|c|c|c|c|c|}
\hline Year & $2011(n=42)$ & $2012(n=33)$ & $2013(n=60)$ & $2014(n=80)$ & $2015(n=94)$ & Total $(n=309)$ \\
\hline FGA & 24 & 32 & 32 & 35 & 26 & 149 \\
\hline SGA & 15 & 18 & 45 & 58 & 57 & 193 \\
\hline Benzodiazepines or other hypnotic & 8 & 10 & 26 & 39 & 27 & 110 \\
\hline Antidepressants & 6 & 4 & 13 & 12 & 21 & 56 \\
\hline Mood stabilisers/anticonvulsants & 4 & 2 & 5 & 6 & 2 & 19 \\
\hline Other medications & 6 & 8 & 10 & 20 & 30 & 74 \\
\hline No medication & 2 & - & 1 & 2 & 3 & 8 \\
\hline No information & 7 & 2 & 1 & 3 & 7 & 20 \\
\hline Mean number of medications & 2.0 & 1.6 & 1.9 & 2.2 & 2.0 & 2.1 \\
\hline
\end{tabular}

FGA, first-generation antipsychotic; SGA, second generation antipsychotic. 


\begin{tabular}{|lcccccc}
\hline Table 4 & Comorbid drug and alcohol misuse & & & \\
Year & $2011(n=42)$ & $2012(n=33)$ & $2013(n=60)$ & $2014(n=80)$ & $2015(n=94)$ & Total $(n=309)$ \\
\hline Drugs & $7(17 \%)$ & $8(24 \%)$ & $19(32 \%)$ & $27(34 \%)$ & $22(23 \%)$ \\
\hline Excess alcohol & $8(19 \%)$ & $9(27 \%)$ & $19(32 \%)$ & $24(30 \%)$ & $13(14 \%)$ \\
\hline
\end{tabular}

Number and percentages of referrals with a history of use of illegal drugs or legal highs declared within records, and number of referrals with significant alcohol-related history, e.g. consistently over weekly recommended allowance, often by a considerable margin.

an acute episode of psychotic illness, supporting the conclusion that IHTT can help people manage an acute psychotic episode, and avoid hospital admission. This belies a negative therapeutic outlook for schizophrenia, with the majority of people with schizophrenia in crisis or relapse avoiding the need for hospital care.

The total CGI score diminished by 1.05 for the whole sample, and a one-point shift in CGI is usually regarded as a clinically significant change. ${ }^{9}$ Since CGI has been shown ${ }^{10}$ to be an effective method of mapping illness severity, with reliability equivalent to that of more complex scales such as Positive and Negative Symptom Scale (PANSS), we can conclude from our results that the Edinburgh IHTT service, on average, can help reduce the severity of schizophrenia and related disorders. The CGI score of those people discharged home from IHTT improved by 2.03 compared with those being transferred to hospital from IHTT, implying an association between hospital admission and a worsening CGI score, and adding to the face validity of this measure. A study from Taiwan ${ }^{11}$ also showed that patients with schizophrenia treated with community home care case management had a significant reduction in the frequency of hospital admission, and a systematic review ${ }^{12}$ from 2017 showed that home-based intervention improves patients' quality of life and autonomy.

Twenty-four per cent of patients were admitted to hospital from IHTT, and the average CGI score at entry to IHTT of those who were eventually admitted to hospital was 0.16 higher than those who were eventually discharged home. This implies that those with a more severe psychotic relapse were more likely to require hospital admission, although non-illness-related factors such as accommodation were not taken into account in this study.

Of note, the gender ratio of the patient population in our study was almost 1:1, whereas usually one would expect a ratio of 1.4:1 in a population of patients with a diagnosis of schizophrenia. ${ }^{5}$ This may have been due to our inclusion criteria being not just schizophrenia but also its related disorders. Additionally, amongst the five deaths in our patient population over the 5-year period there was only one suicide, which is theoretically lower than expected for this city-wide population. ${ }^{8}$ Beyond this, however, we have no reason to regard our study population as unrepresentative, given the lack of local alternative acute care pathways.

The mean number of medications a patient was taking was 2.1, with $21 \%$ of patients prescribed four or more medications. This replicates work elsewhere in the $\mathrm{UK}^{13}$ indicating that polypharmacy in this complex disorder is often the 'real world' norm, despite a paucity of supportive evidence on added efficacy. ${ }^{14,15}$ Polypharmacy is also generally associated with an increased burden of adverse side- effects. ${ }^{16}$ Our results show that people who were taking no medication, or were non-adherent with their medications, were more likely to be admitted to hospital than those taking medication. Interestingly, patients taking four or more medications demonstrated the most improvement, as measured by CGI-I.

Our results showed that $9 \%$ of the patient population in this study were taking no medication or were nonadherent with medication according to recorded clinician ratings, although medication non-adherence rates in wider community samples are closer to $50 \%{ }^{17}$ This discrepancy may have been due to many factors, including simple forgetfulness, a reluctance of the patient to acknowledge nonadherence, and a failure to enquire about it in a consistent manner.

Drug and alcohol misuse are common in people with schizophrenia, ${ }^{18}$ and our results show a higher proportion of people with schizophrenia misusing drugs than alcohol (Table 4), suggesting a correlation between drug use, rather than alcohol misuse, and schizophrenia. Menezes et $a l^{19}$ analysed drug and alcohol problems among 171 individuals with severe mental illness in South London, finding that $36 \%$ had a 1-year prevalence rate for any substance problem, compared with alcohol at $32 \%$ and drugs at $16 \%$, contrary to our findings. This comorbidity has been shown previously to contribute to the premature mortality of those with schizophrenia. $^{20}$

\section{Limitations}

There were various limitations to this study. First, the patient population was from one IHTT only; therefore, the results may not be applicable to all IHTTs or CRHTs. Furthermore, this was an observational study, so comparative conclusions should not be made. Although CGI has been shown to be a good measure of observer rated clinical outcomes, ${ }^{10}$ it remains open to potential bias. Finally, data collection errors may possibly have occurred without our knowledge.

\section{Summary}

Intensive multi-disciplinary home treatment (here via IHTT) can be an effective intervention for most people suffering an acute episode of psychotic illness, with the majority being successfully managed at home and not requiring hospital admission. The minority that do go on to need hospital admission tend to have higher initial severity of illness, as measured by CGI, as well as being on no medication or being non-adherent with medication according to their treating clinicians. 
Polypharmacy appears to be standard medical practice for people with schizophrenia, despite a lack of supportive evidence of added efficacy. Drug and alcohol misuse are common in people with psychotic illness, with higher rates than in the general population, arguably contributing to the significant morbidity and mortality seen with this condition. ${ }^{19}$

\section{About the authors}

Hannah Chu Han Huang is a core trainee at the South London and Maudsley Trust, UK; Mark Taylor is an honorary senior lecturer at the University of Edinburgh, UK, University of Queensland, and an associate professor and consultant psychiatrist at Metro South Health, Brisbane, Australia; Alasdair Carmichael was a medical student at the University of Edinburgh, UK.

\section{Supplementary material}

Supplementary material is available online at https://doi.org/10.1192/bjb. 2018.56

\section{Acknowledgements}

We thank Dr Kader, Julie, Trish and all other IHTT staff, as well as Nicoletta Adamo.

\section{References}

1 Johnson S. Crisis resolution and home treatment teams: an evolving model. Adv Psychiatr Treat 2013; 19(2): 115-23.

2 McCrone P, Johnson S, Nolan F, Pilling S, Sandor A, Hoult J, et al. Economic evaluation of a crisis resolution service: a randomised controlled trial. Epidemiol Psichiatr Soc 2009; 18(1): 54-8.

3 Barker V, Taylor M, Kader I, Stewart K, Le Fevre P. Impact of crisis resolution and home treatment services on user experience and admission to psychiatric hospital. Psychiatrist 2011; 35(3): 106-10.

4 Hopkins C, Niemiec S. Mental health crisis at home: service user perspectives on what helps and what hinders. J Psychiatr Ment Health Nurs. 2007; 14(3): 310-8.

5 Whiteford HA, Degenhardt L, Rehm J, et al. Global burden of disease attributable to mental and substance use disorders: findings from the Global Burden of Disease Study 2010. Lancet 2013; 382(9904): 1575-86.
6 Zipursky RB, Menezes NM, Streiner DL. Risk of symptom recurrence with medication discontinuation in first-episode psychosis: a systematic review. Schizophr Res 2014; 152: 408-414.

7 Ascher-Svanum H, Zhu B, Faries DE, et al. The cost of relapse and the predictors of relapse in the treatment of schizophrenia. BMC Psychiatry 2010; doi: 10.1186/1471-244X-10-2

8 Hor K, Taylor M. Suicide and schizophrenia: a systematic review of rates and risk factors. J Psychopharmacol 2010; 24(4_supplement): 81-90.

9 Guy W. Clinical Global Impressions (CGI) Scale, Modified. In Task Force for the Handbook of Psychiatric Measures. Handbook of Psychiatric Measures (1st ed.) (ed A John). American Psychiatric Association, 2000.

10 Leucht S, Kane JM, Etschel E, Kissling W, Hamann J, Engel RR. Linking the PANSS, BPRS, and CGI: clinical implications. Neuropsychopharmacology 2006; 31(10): 2318-25.

11 Chang L, et al. Psychopathology, rehospitalization and quality of life among patients with schizophrenia under home care case management in Taiwan. Formosan Med Assoc 2013; 112: 208-15.

12 Gomis O, et al. Domiciliary intervention in psychosis: a systematic review. Actas Esp Psiquiatr 2017; 45(6): 290-302.

13 Patel $M X$, Bishara $D$, Jayakumar $S$, et al. Quality of prescribing for schizophrenia: evidence from a national audit in England and Wales. Eur Neuropsychopharmacol 2014; 24(4): 499-509.

14 Langan J, Shajahan P. Antipsychotic polypharmacy: review of mechanisms, mortality and management. Psychiatrist 2010; 34(2): 58-62.

15 Fleischhacker WW, Uchida H. Critical review of antipsychotic polypharmacy in the treatment of schizophrenia. Int J Neuropsychopharmacol 2014; 17(7): 1083-93

16 Young SL, Taylor M, Lawrie SM. 'First do no harm.' A systematic review of the prevalence and management of antipsychotic adverse effects. Psychopharmacol 2015; 29: 353-62.

17 Lacro JP, Dunn LB, Dolder CR, Leckband SG, Jeste DV. Prevalence of and risk factors for medication nonadherence in patients with schizophrenia: a comprehensive review of recent literature. J Clin Psychiatry 2002; 63: 892-909.

18 Hartz SM, Pato CN, Medeiros H, Cavazos-Rehg P, Sobell JL, Genomic Psychiatry Cohort Consortium (GPCC), et al. Comorbidity of severe psychotic disorders with measures of substance use. JAMA Psychiatry 2014; 71(3): 248-54.

19 Menezes PR, Johnson S, Thornicroft G, et al. Drug and alcohol problems among individuals with severe mental illness in south London. $\mathrm{Br} J$ Psychiatry 1996; 168(5): 612-9.

20 Ajetunmobi O, Taylor M, Stockton D, Wood R. Early death in those previously hospitalised for mental healthcare in Scotland: a nationwide cohort study, 1986-2010. BMJ Open 2013; 3(7): e002768. 